\title{
Clinical Results and Prognostic Factors of Arthroscopic Surgeries for Discoid Lateral Menisci Tear: Analysis of 179 Cases with Minimum 2 Years Follow-up
}

\author{
Ji-Yong Ahn, $\mathrm{MD}^{1}$, Tae-Han Kim, $\mathrm{MD}^{2}$, Bong-Sung Jung, $\mathrm{MD}^{2}$, Sang-Ho Ha, $\mathrm{MD}^{2}$, Bum-Sik Lee, $\mathrm{MD}^{3}$, Jong- \\ Won Chung, $\mathrm{MD}^{4}$, Jong-Min Kim, $\mathrm{MD}^{2}$ and Seong-Il Bin, $\mathrm{MD}^{2}$ \\ ${ }^{1}$ Department of Orthopedic Surgery, Seoul Paik Hospital, Inje University College of Medicine, Seoul; ${ }^{2}$ Department of Orthopedic Surgery, Asan Medical Center, \\ University of Ulsan College of Medicine, Seoul; ${ }^{3}$ Department of Orthopedic Surgery, Incheon St. Mary's Hospital, The Catholic University of Korea College of Medicine, \\ Incheon; ${ }^{4}$ Barunmadi Orthopedics, Seongnam, Korea
}

\begin{abstract}
Purpose: The purpose of this study was to evaluate the clinical results and prognostic factors of arthroscopic surgeries for tears of the discoid lateral menisci.

Materials and Methods: From March 1997 to September 2005, 260 patients received arthroscopic surgeries due to discoid lateral menisci tears. Among these patients, 179 knees in 168 patients were followed up for at least 2 years and were enrolled into this study. The following prognostic factors were evaluated: sex, age, symptom duration, and type of meniscal tear. Visual analogue score (VAS), Lysholm score, and Ikeuchi grade were assessed as clinical results of arthroscopic surgeries.

Results: The mean VAS and Lysholm score improved at the last follow-up. According to the Ikeuchi grade, 104 cases were rated as excellent, 51 cases as good, and 23 cases as fair. Male sex ( $\mathrm{p}<0.033$ ), age younger than 20 years ( $\mathrm{p}=0.0474)$, and symptom duration less than 12 months ( $<0.044)$ were good prognostic factors. However, there was no correlation between tear types of discoid lateral menisci and clinical results.

Conclusions: Sex, age, and symptom duration could be significant prognostic factors of arthroscopic surgeries for tears of discoid lateral menisci.
\end{abstract}

Key words: Discoid lateral meniscus, Arthroscopic surgery, Prognostic factor.

\section{Introduction}

The discoid lateral meniscus, first described by Young in 1889 , has been the subject of numerous studies regarding its etiology, classification, diagnosis, and treatment. This anomaly has been reported to be present in the ranges of $1.4 \%$ to $15.5 \%$

Received April 26, 2011; Revised (1st) July 5, 2011;

(2nd) October 10, 2011; Accepted October 17, 2011.

Correspondence to: Seong-Il Bin, MD.

Department of Orthopedic Surgery, Asan Medical Center, University of Ulsan College of Medicine, 88 Olympic-ro 43-gil, Songpa-gu, Seoul 138736, Korea

Tel: +82-2-3010-3530, Fax: +82-2-488-7877

Email: sibin@amc.seoul.kr

This is an Open Access article distributed under the terms of the Creative Commons Attribution Non-Commercial License (http://creativecommons.org/licenses/by-nc/3.0/) which permits unrestricted non-commercial use, distribution, and reproduction in any medium, provided the original work is properly cited. of the world population ${ }^{1)}$. In the study by Ikeuchi ${ }^{2)}$, a discoid lateral meniscus was observed in $16.6 \%$ of all knees examined arthroscopically during a 20 year period. In the study by Kato et al. ${ }^{3)}$, the prevalence was $33.2 \%$ in the 577 lateral menisci obtained from 306 cadavers. Domestically, Seong et al. ${ }^{4)}$ found discoid lateral meniscus in $15.3 \%$ of patients who underwent arthroscopy and Bin et al. ${ }^{5)}$ reported that $15.2 \%$ of patients who underwent arthroscopic meniscectomy had this variant, $67 \%$ of which were torn. Likewise, the incidence is relatively high in Asian countries, including Korea.

The discoid lateral meniscus is susceptible to tear due to the anomalous shape, thickness, and poor vascularization ${ }^{6-9)}$ and tears are mostly asymptomatic, which can result in delays in diagnosis and in treatment. In addition, choosing a proper operative method can be a challenging task because previous studies on meniscectomy provide conflicting treatment outcomes; factors that may influence the postoperative outcomes have been rarely addressed in the literature.

On the prognostic factors of meniscectomy for the discoid lateral meniscus, Vandermeer and Cunningham ${ }^{10)}$ reported that 
duration of symptoms, type of meniscal tear, and length of followup period were not related to clinical outcomes and excellent results were observed in the cases with age under 20 or male gender of the total 25 meniscectomies at a mean follow-up of 54 months. Aglietti et al. ${ }^{1)}$ performed 17 meniscectomies ( 6 total and 11 partial meniscectomies) for the discoid lateral menisci in patients, who were between 5 to 18 years of age, and found that the type of discoid lateral menisci classified according to Watanabe criteria and type of meniscectomy had no correlation with clinical and radiologic results for a 10-year follow-up period. In this study, we investigated possible prognostic factors of arthroscopic surgery for the discoid lateral meniscus tear and conducted a review of the relevant literature. Our hypothesis for this study was that preoperative factors including gender, age, duration of symptoms, and type of meniscal tear would affect the results of arthroscopic surgeries of the discoid lateral menisci.

\section{Materials and Methods}

Between March 1997 and September 2005, 260 patients underwent arthroscopic surgeries for the discoid lateral menisci at our institution. Among them, 168 patients (179 cases) with a minimum follow-up of 2 years were included in this study. There were 77 males and 102 females with a mean age of 35 years (range, 5 to 70 years). The mean duration from symptom onset to surgery was 29.2 months (range, 1 to 300 months). The mean follow-up period was 48.9 months (range, 24 to 113 months). Total meniscectomy was performed in 16 cases, subtotal meniscectomy in 30 cases, partial meniscectomy in 123 cases, meniscal repair in 1 case. Partial meniscectomy and meniscal repair was done in 3 cases, only arthroscopic examination in 6 cases.

The medical history taken preoperatively and at the last follow-up and intraoperative arthroscopic images were used for assessment in all cases. Telephone interviews were conducted additionally in 72 cases for which the medical records included ambiguous information. For clinical assessment, preoperative, and postoperative Visual Analogue Scale (VAS) score for pain, Lysholm knee score, and Ikeuchi grade ${ }^{2)}$ were compared.

Pain was assessed using a 10-point VAS. For more detailed evaluation, we assessed VAS scores for pain during 5 different activities that can cause pain in patients with a meniscal tear, which included walking, stair climbing, squatting, sitting crosslegged, and sitting to standing or standing to sitting. The total sum of the values was used as a VAS score for pain.

The discoid lateral menisci were categorized according to
Watanabe's classification into complete, incomplete, and Wrisberg types. Type of meniscal tear was classified into radial, horizontal, oblique, complex, degenerative, and longitudinal tears. The bucket handle tear and flap tear were classified into longitudinal tear and oblique tear, respectively.

The patients were divided according to age into 3 groups $(<20$ years, 20-40 years, and $>40$ years) and duration of symptoms into 2 groups ( $<12$ months and $>12$ months). In the absence of studies that provide definite criteria for chronicity, we used 12 months as a criterion based on previous studies ${ }^{11-13)}$, where 6 months or 12 months were used as a criterion.

The relationship between gender and clinical result was analyzed using the independent sample $t$ test and Fisher's exact test. The relationships among age, type of meniscal tear, and clinical results were analyzed using the chi square test and for comparisons among age groups, logistic regression analysis was performed. The relationship between the duration from symptom onset to surgery and clinical result was analyzed using Fisher's exact test. Statistical significance was set at $\mathrm{p}<0.05$.

\section{Results}

The mean VAS score for pain was improved from 26 preoperatively to 12 postoperatively. The mean Lysholm score increased from 72.3 preoperatively to 88.7 postoperatively. The postoperative Ikeuchi grade was excellent in 104 cases, good in 51 , and fair in 23 , and there was no poor case.

The preoperative range of motion was normal in 119 cases. Minor flexion contracture $\left(5^{\circ}-30^{\circ}\right)$ was observed in 60 cases.

The type of discoid lateral meniscus observed during arthroscopic surgery was complete in 87 cases, incomplete in 92 cases, and a Wrisberg-type meniscus was not observed. The type

Table 1. The Distribution of Tear Patterns according to Watanabe's Classification

\begin{tabular}{lccc}
\hline Tear pattern & Incomplete & Complete & Total \\
\hline Longitudinal & 17 & 33 & 50 \\
Radial & 25 & 3 & 28 \\
Degenerative & 8 & 1 & 9 \\
Horizontal & 9 & 30 & 39 \\
Oblique & 2 & 0 & 2 \\
Complex & 28 & 17 & 45 \\
No tear & 1 & 2 & 3 \\
Worn out & 2 & 1 & 3 \\
Total & 92 & 87 & 179 \\
\hline
\end{tabular}


Table 2. The Relation between Tear Pattern and Ikeuchi Grade

\begin{tabular}{llcc}
\hline & \multicolumn{3}{c}{ The knee rating scale of Ikeuchi } \\
\cline { 2 - 4 } & Fair & Good & Excellent \\
\hline Longitudinal & $3(6)$ & $14(28)$ & $33(66)$ \\
Radial & $7(25)$ & $5(17.9)$ & $16(57.1)$ \\
Degenerative & $2(22.2)$ & $1(11.1)$ & $6(66.7)$ \\
Horizontal & $3(7.7)$ & $15(38.5)$ & $21(53.8)$ \\
Oblique & $0(0)$ & $1(50)$ & $1(50)$ \\
Complex & $7(15.6)$ & $14(31.1)$ & $24(53.3)$ \\
No tear & $0(0)$ & $0(0)$ & $3(100)$ \\
Worn out & $1(33.3)$ & $1(33.3)$ & $1(33.3)$ \\
\hline
\end{tabular}

Values are presented number (\%).

No statistically significant difference by chi-square test: $\mathrm{p}=0.3610$.

of meniscal tear was radial in 28 cases, horizontal in 39, oblique in 2, complex in 45, degenerative in 9, and longitudinal in 50, including 38 cases of bucket handle tears. No tear was observed in 3 cases with symptoms. The lateral meniscus was almost completely worn down in 3 cases (Table 1).

Total meniscectomy was performed in 16 cases, subtotal meniscectomy in 30, partial meniscectomy in 123, partial meniscectomy with repair in 3 , and arthroscopic examination only in 6 . Among the 50 longitudinal tears, repair only and partial meniscectomy with repair were performed in 1 case and 3 cases, respectively, because repair seemed to be appropriate given the tear site and degree of degenerationat tear margin. In the remaining 46 cases, repair was not indicated because of prominent degeneration at the periphery of the tear.

Arthroscopic assessment was not followed by treatment in 6 cases. In 3 of the cases, a tear was not observed during a thorough examination using a probe, although pain was present and the range of movement was limited. In the remaining 3 cases, the meniscus was so severely worn down that additional meniscectomy was unnecessary.

The chi square test revealed no significant relationship between the type of meniscal tear and clinical results $(\mathrm{p}=0.3610)$ (Table 2).

On the relationship between gender and clinical results analyzed using the independent sample $t$ test, the postoperative VAS score for pain was significantly different between males and females $(p<0.033)$. The mean score was lower in males $(10.09 \pm 7.906)$ than in females (12.95 \pm 9.415$)$.

The chi square test revealed significant differences in postoperative Ikeuchi grade among the 3 groups divided according to age $(\mathrm{p}=0.0474)$ (Table 3). For more refined comparisons, a logistic regression analysis was performed. Excellent and good grades were referred to as satisfactory results and fair and poor grades
Table 3. The Relation between Age and Postoperative Ikeuchi Grade

\begin{tabular}{lcccc}
\hline & \multicolumn{3}{c}{ Age (yr) } & Total \\
\cline { 2 - 4 } & $<20$ & $20-40$ & $>40$ & \\
\hline Excellent & $24(54.6)$ & $38(69.1)$ & $43(53.7)$ & 105 \\
Good & $18(40.9)$ & $10(18.2)$ & $23(28.8)$ & 51 \\
Fair & $2(4.5)$ & $7(12.7)$ & $14(17.5)$ & 23 \\
\hline
\end{tabular}

Values are presented number (\%).

Statistically significant difference by chi-square test: $\mathrm{p}=0.0474$.

Table 4. The Relation between Duration of Symptom and Postoperative Ikeuchi Grade

\begin{tabular}{lcrc}
\hline \multirow{2}{*}{ Duration of symptoms } & \multicolumn{3}{c}{ The knee rating scale of Ikeuchi } \\
\cline { 2 - 4 } & Fair and good & Excellent & Total \\
\hline Under 12 months & $23(31.5)$ & $50(68.5)$ & 73 \\
Over 12 months & $51(48.1)$ & $55(51.9)$ & 106 \\
Total & $74(41.3)$ & $105(58.7)$ & 179 \\
\hline
\end{tabular}

Values are presented number (\%).

Statistically significant difference by Fisher's exact test: $\mathrm{p}<0.044$.

as unsatisfactory results for comparison. The odd of obtaining satisfactory results was 4.455 times higher in the $<20$-years-old group compared to the $\geq 40$ years old group $(\mathrm{p}=0.05$ ).

The mean duration from symptom onset to surgery was 29.2 months. On the comparison between the 2 groups divided according to the duration of symptoms, the percentage of cases with an excellent Ikeuchi grade after surgery was $68.5 \%$ in the group with $<12$ months of duration of symptoms and $51.9 \%$ in the other group with $\geq 12$ months of duration of symptoms. According to the Fisher's exact test, the percentage of cases with an excellent Ikeuchi grade was significantly higher in the group with $<12$ months of duration of symptoms $(\mathrm{p}<0.044)$ (Table 4$)$.

\section{Discussion}

The purpose of this study was to assess the influence of preoperative factors including gender, age, duration of symptoms, and type of meniscal tear on the arthroscopic treatment results of the discoid lateral meniscus. We found that the clinical results were significantly better in the patients who were male and $\leq 20$ years of age and had $<12$ months' duration of symptoms. No significant relationship was found between the type of meniscal tear and clinical results.

Our findings were consistent with those of the study by Vandermeer and Cunningham ${ }^{10)}$ in that excellent results were related to male gender and $\leq 20$ years of age, although partial meniscectomy was the treatment of choice in all cases in their 
study, whereas we performed total/subtotal meniscectomy or repair as well as partial meniscectomy. On the other hand, duration of symptoms was not related to postoperative results in their study.

Asik et al. ${ }^{14)}$ found that degenerative tears, complex tears, and preoperative radiological degenerative changes in lateral compartment were associated with poor clinical results in 197 patients who had undergone partial meniscectomy for symptomatic discoid lateral meniscus and had been available for a mean follow-up of 57.8 months. On the other hand, they reported that gender, age, and duration of symptoms had no influence on the clinical results, contrary to our study.

However, Okazaki et al. ${ }^{15)}$ reported that patients who were $\leq 25$ years of age obtained significantly better clinical results during a mean follow-up of 16 years after subtotal meniscectomy for lateral discoid lateral meniscus in 29 cases. Wong and Wang ${ }^{13)}$ assessed 32 cases of partial/subtotal meniscectomy and repair for discoid lateral meniscus for a mean follow-up of 52 months and reported that surgical technique and preoperative anatomical lower limb alignment were not related to the clinical results, whereas age correlated with clinical outcome. Therefore, we believe that there are more studies that corroborate than refute our findings that age is associated with clinical results of treatment for the discoid lateral meniscus.

Arthroscopic treatment results for normal meniscus tears, albeit not discoid lateral meniscus tears, have been documented in many studies regarding the relationship between age, gender, and duration of symptoms and clinical results. In the study by Aglietti et al ${ }^{16)}$ where 100 cases of partial meniscectomy followed for a mean of 3.5 years, males were found to have obtained better results than females, but age did not yield statistically significant differences. Bolano and Grana ${ }^{11}$ analyzed $\geq 5$ years of follow-up results of arthroscopic partial meniscectomy in 50 patients and reported that factors associated with satisfactory results were age less than 40 years, symptoms less than 12 months of duration, degenerative tear, and chondromalacia less than grade II. According to Wouters et al. ${ }^{17)}, \geq 12$ months' duration of symptom was associated with unsatisfactory results of arthroscopic meniscectomy and age had no impact on the postoperative results for $\geq 33$ months of follow-up period in 57 patients, who were $\geq 50$ years of age. The overall results of the study by Scheller et al. ${ }^{12)}$ were similar to ours. They reported that $\geq 6$ month duration of symptoms and advanced age were related to poor results in 75 cases of partial meniscectomy for lateral meniscus tear with a mean follow-up of 12.3 years.

However, Bonamo et al. ${ }^{18)}$ reported that although the results tended to be worse in females than males, and in patients who were $\geq 60$ years old, age and gender had no statistically significant relationship with the results in 118 patients, who were $\geq 40$ years of age and followed for a mean of 3.3 years. Katz et al. ${ }^{19)}$ and Bonneux and Vandekerckhove ${ }^{20)}$ assessed the relationship between gender and results and Burks et al. ${ }^{21)}$ and Higuchi et al. ${ }^{22)}$ investigated the influence of age and gender on the results. In these studies, no statistically significant relationship was found between the parameters and results. Likewise, there are many studies with results that are contradictory to ours.

There could be some limitations in comparing the current study with these studies where normal menisci, not the discoid lateral menisci, were the subject, medial/lateral menisci were not separated for analyses, and partial meniscectomy was the major treatment modality. However, we believe that prognostic factors of arthroscopic surgery for meniscal tears would be similar for the normal meniscus and the discoid lateral meniscus.

Regarding the factors, we associated with clinical results including gender, age, and duration of symptoms, there are more studies that are consistent with our findings than those providing contrary results. In particular, the significance of our study can be found in the fact that our results were based on the assessment of a large number of discoid lateral menisci.

One of the limitations of our study is that radiological changes were not included in the analysis. In addition, the relationship between surgical technique and results was not investigated, although we performed several arthroscopic surgical methods. Considering that there is an ongoing debate on the proper surgical method for discoid lateral meniscus tears, the influence of various surgical methods on the clinical and radiological results should be investigated in further studies involving a larger number of patients.

\section{Conclusions}

We believe that male gender, $\leq 20$ years of age, and $\leq 12$ months' duration of symptom can be associated with favorable clinical results of arthroscopic surgery for discoid lateral meniscus.

\section{References}

1. Aglietti P, Bertini FA, Buzzi R, Beraldi R. Arthroscopic meniscectomy for discoid lateral meniscus in children and adolescents: 10-year follow-up. Am J Knee Surg. 1999;12:837.

2. Ikeuchi H. Arthroscopic treatment of the discoid lateral 
meniscus. Technique and long-term results. Clin Orthop Relat Res. 1982;(167):19-28.

3. Kato Y, Oshida M, Aizawa S, Saito A, Ryu J. Discoid lateral menisci in Japanese cadaver knees. Mod Rheumatol. 2004; 14:154-9.

4. Seong SC, Cho SH, Chung MS, Park YS. The study on the menisci of Korean adults. J Korean Orthop Assoc. 1984;19: 1061.

5. Bin SI, Kim JC, Kim JM, Park SS, Han YK. Correlation between type of discoid lateral menisci and tear pattern. Knee Surg Sports Traumatol Arthrosc. 2002;10:218-22.

6. Arnoczky SP, Warren RF. The microvasculature of the meniscus and its response to injury. An experimental study in the dog. Am J Sports Med. 1983;11:131-41.

7. Bohr H. On the diagnosis of the meniscus lesion in the knee with special regard to the value of arthrography. Acta Orthop Scand. 1959;29:146-9.

8. Hayashi LK, Yamaga H, Ida K, Miura T. Arthroscopic meniscectomy for discoid lateral meniscus in children. J Bone Joint Surg Am. 1988;70:1495-500.

9. Rohren EM, Kosarek FJ, Helms CA. Discoid lateral meniscus and the frequency of meniscal tears. Skeletal Radiol. 2001;30:316-20.

10. Vandermeer RD, Cunningham FK. Arthroscopic treatment of the discoid lateral meniscus: results of long-term followup. Arthroscopy. 1989;5:101-9.

11. Bolano LE, Grana WA. Isolated arthroscopic partial meniscectomy. Functional radiographic evaluation at five years. Am J Sports Med. 1993;21:432-7.

12. Scheller G, Sobau C, Bulow JU. Arthroscopic partial lateral meniscectomy in an otherwise normal knee: clinical, functional, and radiographic results of a long-term follow-up study. Arthroscopy. 2001;17:946-52.

13. Wong T, Wang CJ. Functional analysis on the treatment of torn discoid lateral meniscus. Knee. 2011;18:369-72.

14. Asik M, Sen C, Taser OF, Alturfan AK, Sozen YV. Discoid lateral meniscus: diagnosis and results of arthroscopic treatment. Knee Surg Sports Traumatol Arthrosc. 2003;11: 99-104.

15. Okazaki K, Miura H, Matsuda S, Hashizume M, Iwamoto Y. Arthroscopic resection of the discoid lateral meniscus: longterm follow-up for 16 years. Arthroscopy. 2006;22:967-71.

16. Aglietti P, Buzzi R, Bassi PB. Arthroscopic partial meniscectomy in the anterior cruciate deficient knee. Am J Sports Med. 1988;16:597-602.

17. Wouters E, Bassett FH 3rd, Hardaker WT Jr, Garrett WE Jr. An algorithm for arthroscopy in the over-50 age group. Am J Sports Med. 1992;20:141-5.

18. Bonamo JJ, Kessler KJ, Noah J. Arthroscopic meniscectomy in patients over the age of 40. Am J Sports Med. 1992;20:4228.

19. Katz JN, Harris TM, Larson MG, Krushell RJ, Brown CH, Fossel AH, Liang MH. Predictors of functional outcomes after arthroscopic partial meniscectomy. J Rheumatol. 1992;19:1938-42.

20. Bonneux I, Vandekerckhove B. Arthroscopic partial lateral meniscectomy long-term results in athletes. Acta Orthop Belg. 2002;68:356-61.

21. Burks RT, Metcalf MH, Metcalf RW. Fifteen-year followup of arthroscopic partial meniscectomy. Arthroscopy. 1997;13:673-9.

22. Higuchi H, Kimura M, Shirakura K, Terauchi M, Takagishi K. Factors affecting long-term results after arthroscopic partial meniscectomy. Clin Orthop Relat Res. 2000;(377):161-8. 